\title{
Trivium
}

Revue franco-allemande de sciences humaines et sociales - Deutsch-französische Zeitschrift für Geistesund Sozialwissenschaften

15 | 2013

La science pense en plusieurs langues

\section{Penser en langues}

Présentation du Vocabulaire Européen des philosophies

\section{Barbara Cassin}

\section{OpenEdition}

\section{Journals}

Édition électronique

URL : http://journals.openedition.org/trivium/4768

DOI : $10.4000 /$ trivium.4768

ISSN : 1963-1820

\section{Éditeur}

Les éditions de la Maison des sciences de l'Homme

\section{Référence électronique}

Barbara Cassin, «Penser en langues », Trivium [En ligne], 15 | 2013, mis en ligne le 09 décembre 2013 consulté le 08 septembre 2020. URL : http://journals.openedition.org/trivium/4768 ; DOI : https:// doi.org/10.4000/trivium.4768

Ce document a été généré automatiquement le 8 septembre 2020

\section{(c) $(1) \&$}

Les contenus des la revue Trivium sont mis à disposition selon les termes de la Licence Creative Commons Attribution - Pas d'Utilisation Commerciale - Pas de Modification 4.0 International. 


\title{
Penser en langues
}

\author{
Présentation du Vocabulaire Européen des philosophies
}

\author{
Barbara Cassin
}

\section{NOTE DE L'ÉDITEUR}

Ce texte a été modifié par son auteur en vue de sa publication dans le présent dossier thématique.

1 L'un des problèmes les plus urgents que pose l'Europe est celui des langues. On peut envisager deux types de solution: choisir une langue dominante, dans laquelle se feront désormais les échanges - un anglo-américain mondialisé ; ou bien jouer le maintien de la pluralité, en rendant manifeste à chaque fois le sens et l'intérêt des différences, seule manière de faciliter réellement la communication entre les langues et les cultures. Le Vocabulaire européen des philosophies, Dictionnaire des intraduisibles s'inscrit dans la seconde optique. Mais il regarde vers l'avenir plutôt que vers le passé : il n'est pas lié à une Europe rétrospective et chosifiée - laquelle d'ailleurs ? -, définie par un cumul d'héritages juxtaposés qui renforcerait les particularismes, mais à une Europe en cours, en activité, energeia plutôt que ergon, qui travaille les écarts, les tensions, les transferts, les appropriations, les contresens, pour mieux se fabriquer.

2 Le point de départ est une réflexion sur la difficulté de traduire en philosophie. Nous avons voulu penser la philosophie en langues, traiter les philosophies comme elles se disent, et voir ce que cela change dans nos manières de philosopher. C'est pourquoi nous n'avons pas confectionné un énième Dictionnaire ou Encyclopédie de la philosophie, traitant pour eux-mêmes des concepts, des auteurs, des courants et des systèmes, mais un Vocabulaire européen des philosophies, qui part de mots pris dans la différence commensurable des langues, du moins des principales langues dans lesquelles s'est écrit de la philosophie en Europe - après Babel. De ce point de vue, le Vocabulaire des institutions indo-européennes d'Émile Benveniste est l'ouvrage, pluraliste et comparatiste, qui nous a servi de modèle : pour trouver le sens d'un mot dans une langue, il met au jour les réseaux dans lesquels il s'insère et cherche à comprendre 
comment un réseau fonctionne dans une langue en le rapportant aux réseaux d'autres langues.

3 Nous n'avons pas travaillé sur tous les mots, ni sur toutes les langues eu égard à un mot, et encore moins sur toutes les philosophies. Nous avons pris pour objet des symptômes de différence, les "intraduisibles", entre un certain nombre de langues européennes d'aujourd'hui, en régressant aux langues anciennes (grec, latin) et en passant par l'hébreu, par l'arabe, chaque fois que c'était nécessaire à l'intelligibilité de ces différences. Parler d'intraduisibles n'implique nullement que les termes en question, ou les expressions, les tours syntaxiques et grammaticaux, ne soient pas traduits et ne puissent pas l'être - l'intraduisible, c'est plutôt ce qu'on ne cesse pas de (ne pas) traduire. Mais cela signale que leur traduction, dans une langue ou dans une autre, fait problème, au point de susciter parfois un néologisme ou l'imposition d'un nouveau sens sur un vieux mot : c'est un indice de la manière dont, d'une langue à l'autre, tant les mots que les réseaux conceptuels ne sont pas superposables - avec mind, entend-on la même chose qu'avec Geist ou qu'avec esprit; pravda, est-ce justice ou vérité, et que se passe-t-il quand on rend mimesis par imitation? Chaque entrée part ainsi d'un nœud d'intraductibilité, et procède à la comparaison de réseaux terminologiques, dont la distorsion fait l'histoire et la géographie des langues et des cultures. Le Vocabulaire européen des philosophies est un «dictionnaire des intraduisibles » en ce qu'il explicite dans son domaine les principaux symptômes de différence des langues.

4 La sélection des entrées résulte d'un double travail d'exploration, diachronique et synchronique. La diachronie, pour réfléchir aux passages, aux transferts et aux bifurcations: du grec au latin, du latin antique au latin scolastique, puis humaniste, avec les moments d'interaction avec la tradition juive et la tradition arabe; d'une langue ancienne à une langue vernaculaire; d'une langue vernaculaire à une autre; d'une tradition, d'un système et d'un idiome philosophique à d'autres; d'un champ du savoir et d'une logique disciplinaire à d'autres. On retrouve ainsi l'histoire des concepts, en dégageant les tournants, les fractures et les opérateurs qui déterminent une "époque». La synchronie, pour constituer un état des lieux, en arpentant l'actualité des paysages philosophiques nationaux; on se trouve confronté à l'irréductibilité des oublis et des inventions: apparitions sans équivalent, intrus, doublets, cases vides, faux amis, contresens, qui marquent dans une langue la cristallisation de thèmes et la spécification d'une opération. On se demande alors, à partir des œuvres modernes qui sont à la fois causes et effets de l'état philosophique d'une langue donnée, pourquoi des termes qu'on considère d'ordinaire comme immédiatement équivalents n'ont ni le même sens ni le même champ d'application - ce que peut une pensée dans ce que peut une langue.

5 L'espace européen a été dès le départ le cadre de notre travail. Le Vocabulaire a de fait une ambition politique: faire en sorte que les langues de l'Europe soient prises en compte, et pas seulement d'un point de vue patrimonial comme on préserve les espèces menacées. À cet égard, il y a deux positions dont nous nous démarquons clairement. La première, c'est le tout anglais, ou plutôt le " tout-à-l'anglais » - cet anglais officiel de la communauté européenne et des colloques scientifiques, qui fonctionne certes, mais qui n'est presque plus une langue (les «vrais Anglais » sont ceux qu'on a le plus de mal à comprendre). L'anglais s'est aujourd'hui imposé comme "langue internationale auxiliaire » pour reprendre une expression d'Umberto Eco, il a pris place dans la série chronologique des langues véhiculaires (le grec, le latin, le français) : c'est à la fois la 
langue universelle de la technocratie cultivée et la langue du marché, nous en avons besoin, pour le meilleur comme pour le pire. Mais la situation philosophique de l'anglais comme langue unique mérite qu'on l'examine un peu autrement. Cette fois, l'anglais est plutôt dans la ligne de la caractéristique universelle rêvée par Leibniz. Non que la langue anglaise puisse jamais se réduire à un calcul conceptuel sur le modèle des mathématiques : c'est, comme toute autre, une langue naturelle, c'est-à-dire une langue de culture, magnifique et forte de toutes ses idiosyncrasies. Cependant, pour une certaine tendance de la "philosophie analytique " (il est vrai qu'aucune précaution terminologique ne sera jamais suffisante ici, puisque l'étiquette vaut, via le linguistic turn, pour ceux-là mêmes qui nous réapprennent à interroger le langage, de Wittgenstein à Austin, Quine ou Cavell), la philosophie relève seulement d'un universel logique, identique en tous temps et en tous lieux - Aristote, mon collègue à Oxford. Peu importe alors la langue qui habille le concept, en l'occurrence l'anglais. Cette première motivation universaliste vient en croiser une seconde. Toute la tradition anglo-saxonne s'est attachée à ne pas jargonner, à refuser le langage ésotérique, à dégonfler les baudruches de la métaphysique : l'anglais se présente, cette fois dans sa singularité de langue, comme celle du common sense et de l'expérience commune, y compris l'expérience commune de la langue. L'angélisme du rationnel et le militantisme du langage ordinaire se rejoignent pour étayer une prévalence de l'anglais, qui se traduit, dans le pire des cas, par le déni du statut de philosophie à cette philosophie continentale engluée dans les contingences de l'histoire et des langues.

$\mathrm{Ni}$... ni ... L'autre position dont nous nous démarquons est celle qui mène du génie des langues, avec tous ses clichés, au "nationalisme ontologique» (c'est cette fois une expression de Jean-Pierre Lefebvre). On la trouve imagée par Herder, au moment où il fait de la traduction, comme imitation et transplantation, la vocation propre de la langue allemande :

" alors qu'en Italie la muse converse en chantant, qu'en France elle raconte et ratiocine avec préciosité, qu'en Espagne elle a l'imagination chevaleresque, qu'en Angleterre, elle pense avec acuité et profondeur, que fait-elle en Allemagne? Elle imite. Imiter serait ainsi son caractère [...] A cette fin, nous avons en notre pouvoir un admirable moyen, notre langue; elle peut être pour nous ce qu'est la main pour l'homme imitateur d'art » (J.G. Herder, Lettres sur l'avancement de l'humanité, in : P. Caussat, D. Adamski, M. Crépon : La Langue source de la nation, Mardaga, 1996, trad. de P. Caussat, p. 105).

Elle est représentée par une certaine tradition heideggerienne de "La " langue philosophique, c'est-à-dire la mieux à même de dire fidèlement l'être, qui occupe une place prédominante dans cette histoire de la philosophie occidentale si continentale. Martin Heidegger considère que la pensée occidentale naît moins en Grèce qu'en grec, et que seule la langue allemande se hausse au niveau du grec dans la hiérarchie des langues philosophantes, si bien que « l'intraductibilité devient à la limite le critère du vrai » (Jean-Pierre Lefebvre, «Philosophie et philologie: les traductions des philosophes allemands », in : Encyclopaedia universalis, Symposium, Les Enjeux, 1, 1990, p. 170).

«La langue grecque est philosophique, autrement dit [...] philosophait elle-même déjà en tant que langue et que configuration de langue. Et autant vaut de toute langue authentique, naturellement à des degrés divers. Ce degré se mesure à la profondeur et à la puissance de l'existence d'un peuple et d'une race qui parle la langue et existe en elle. Ce caractère de profondeur et de créativité philosophique de la langue grecque, nous ne le retrouvons que dans notre langue allemande » 
(M. Heidegger : De l'essence de la liberté humaine, Introduction à la philosophie [1930], tr.

E. Martineau, Gallimard, 1987, p. 57 s.).

Même si c'est en un sens « vrai » (des vocables et des tournures du grec et de l'allemand sont des point de passage obligés dans beaucoup d'articles du dictionnaire), ce n'est pas cette vérité-là qu'il nous faut. Notre travail est au plus loin d'une telle sacralisation de l'intraduisible, fondée sur l'idée d'une incommensurabilité absolue des langues et liée à la quasi-sainteté de certaines langues. C'est pourquoi, à l'écart d'une histoire de la philosophie téléologique vectorisée selon le registre du gain ou de la perte, nous n'avons conféré à aucune langue, morte ou vivante, de statut particulier.

7 Ni universalisme logique indifférent aux langues, ni nationalisme ontologique avec essentialisation du génie des langues : face à ces deux positions, quelle est la nôtre ? Si je devais la caractériser, je parlerais deleuzien : "déterritorialisation ». Elle joue la géographie contre l'histoire, le réseau sémantique contre le concept isolé. Nous sommes partis du multiple (les pluriels l'indiquent: vocabulaire des philosophies, dictionnaire des intraduisibles), et pour y demeurer : nous avons instruit la question de l'intraduisible sans viser l'unité, qu'on la place à l'origine (langue source, mots fontaines, fidélité à la donation ontologique) ou à la fin (langue messianique, communauté rationnelle).

Multiplicité des langues d'abord - comme le souligne Wilhelm von Humboldt, «le langage se manifeste dans la réalité uniquement comme multiplicité " (Über die Verschiedenheiten..., in Gesammelte Schriften, ed. A. Leitzmann et al., Berlin, Behr, vol. VI, p. 240). Babel est une chance, à condition de comprendre que « la pluralité des langues est loin de se réduire à une pluralité de désignations d'une chose : elles sont différentes perspectives de cette même chose et quand la chose n'est pas l'objet des sens externes, on a affaire souvent à autant de choses autrement façonnées par chacun » («Fragment de monographie sur les Basques » [1822], dans P. Caussat : La langue source de la nation, Mardaga, 1996, p. 433). Les perspectives sont constitutives de la chose, chaque langue est une vision du monde qui attrape un autre monde dans son filet, qui performe un monde, et le monde commun est moins un point de départ qu'un principe régulateur. Schleiermacher met parfaitement en lumière la tension qui existe entre un concept, dans sa prétention à l'universalité, et son expression linguistique, lorsqu'il affirme qu'en philosophie, plus que dans n'importe quel domaine,

« chaque langue contient [...] un système de concepts qui, précisément parce qu'ils se touchent, s'unissent et se complètent dans la même langue, forment un tout dont les différentes parties ne correspondent à aucune de celles du système des autres langues, à l'exception et encore, de Dieu et de l'Être, le premier substantif et le premier verbe. Car même l'absolument universel, bien qu'il se trouve hors du domaine de la particularité, est éclairé et coloré par la langue » (Des différentes méthodes du traduire, tr. de A. Berman, Le Seuil (Points-Bilingues), 1999, p. 84-85).

C'est le « et encore » qu'il faut souligner : même Dieu et l'Être sont éclairés et colorés par la langue ; l'universalité des concepts est entièrement absorbée dans la singularité linguistique.

9 La multiplicité n'est pas seulement entre les langues, mais en chaque langue. Une langue, telle que nous l'avons considérée, n'est pas un fait de nature, un objet, mais un effet pris dans l'histoire et la culture, et qui ne cesse de s'inventer - derechef, energeia plutôt qu'ergon. Si bien que l'objet du dictionnaire est constitué par les langues en leurs œuvres, et par les traductions de ces œuvres en différentes langues, à différentes époques. Les réseaux de mots et de sens que nous avons cherché à penser sont des 
réseaux d'idiomes philosophiques datables, mis en place par des auteurs spécifiques, dans des écrits particuliers; ce sont des réseaux singuliers et ponctuels, liés à l'adresse (exotérique ou ésotérique), au niveau de langue, au style, au rapport à la tradition (modèles, références, palimpsestes, ruptures, innovations). Tout auteur, et le philosophe est un auteur, en même temps qu'il écrit dans une langue, fabrique sa langue - comme dit Schleiermacher du rapport entre un auteur et sa langue : « il est son organe et elle est le sien» ("L'herméneutique générale, 1809-1810», in : Herméneutique, trad. C. Berner, Cerf/PUL, 1987, 15, p. 75). L'intraduisible est donc aussi de l'ordre du cas par cas.

10 La multiplicité est enfin celle des sens d'un mot dans une langue donnée. Comme dit Jacques Lacan dans L'Étourdit: "Une langue, entre autres, n'est rien de plus que l'intégrale des équivoques que son histoire y a laissé persister » (Scilicet, 4, Le Seuil, 1973, p. 47). Le Vocabulaire nous a conduit à interroger le phénomène de l'homonymie (même mot, plusieurs définitions: le chien, constellation céleste et animal aboyant) dont l'homophonie (le vair et le verre) n'est qu'un cas extrême et une caricature moderne. On sait depuis Aristote et son analyse du verbe « être » qu'il n'est pas si facile de distinguer entre homonymie et polysémie : le sens d'un mot, meaning en anglais, le sens du toucher, sense, le sens d'un cours d'eau, direction, voilà une trace équivoque de la polysémie du latin sensus, lui-même traduction du grec nous (flair, esprit, intelligence, intention, intuition etc.) bien autrement polysémique de notre point de vue. La variation d'une langue à l'autre rend sensible à ces distorsions et à ces flux sémantiques; elle permet d'instruire les équivoques dont chaque langue est porteuse, leur signification, leur histoire, leur croisement avec celles des autres langues.

11 Dans son «Introduction" à l'Agamemnon d'Eschyle, qu'il considère comme « intraduisible », Humboldt suggère qu'il faudrait réaliser un ouvrage qui étudie la « synonymie des langues » et prenne acte du fait que chaque langue exprime le concept avec une différence :

«Un mot est si peu le signe d'un concept que le concept ne peut même pas naître sans lui, encore moins être fixé ; l'action indéterminée de la force de pensée se condense dans un mot comme de légers nuages apparaissent dans un ciel pur». "Une telle synonymie des langues principales n'a encore jamais été tentée ", ajoute-t-il, «bien qu'on en trouve chez beaucoup d'écrivains des fragments, mais elle deviendrait, si elle est traitée avec esprit, un ouvrage des plus séduisants " (tr. de D. Thouard: Sur le caractère national des langues et autres écrits sur le langage, Seuil, 2000, p. 33-35).

Cet « ouvrage des plus séduisants ", c'est peut-être bien notre Vocabulaire. J'espère qu'il rendra sensible une autre manière de philosopher, qui ne pense pas le concept sans le mot, car il n'y a pas de concept sans mot.

Le Vocabulaire a l'ambition de constituer une cartographie des différences philosophiques européennes en capitalisant le savoir des traducteurs, et de ces traducteurs (historiens, exégètes, critiques, interprètes) que nous sommes en tant que philosophes. C'est un instrument de travail d'un type nouveau, indispensable à la communauté scientifique élargie qui cherche à se constituer, en même temps qu'un guide de l'Europe philosophique pour les étudiants, les enseignants, les chercheurs, les curieux de leur langue et de celle des autres. Ce travail vraiment collectif (long, difficile, frustrant, à refaire, à poursuivre) a en tout cas séduit chacun de nous, l'a poussé à remettre sur le métier et à reconsidérer selon d'autres perspectives ce qu'il croyait savoir en philosophie, de la philosophie 


\section{Les traductions-transferts du Vocabulaire - ce que veut une langue}

13 L'enjeu se trouve redoublé avec la traduction, ou plutôt les traductions, du Vocabulaire. Elles font prendre conscience qu'il ne s'agit en aucun cas d'un simple transport de contenu d'une langue à une autre, éventuellement automatisable; mais bien d'une adaptation et d'une réinvention, dépendant de ce que veulent une langue et une culture à un moment donné de leur histoire - bref d'un vecteur de politique intellectuelle, et de politique tout court.

14 Il ne saurait s'agir en effet d'une traduction mécanique. Le Vocabulaire a pensé les «intraduisibles» au sein d'un espace certes international et plurilingue, mais néanmoins francophone, au sens strict de parlant français, et il les a décrits au moyen $\mathrm{du}$ français comme métalangue. Toute traduction dans une autre langue doit donc d'abord faire la part entre les entrées qui sont en français "générique » ou "métalinguistique », et celles qui sont en français "idiomatique ». On peut entendre cette différence en comparant, par exemple, les deux articles " Aimer, amour, amitié » et « Nostalgie ». " Aimer » tient lieu, en français, de tout un pan sémantique analysable différentiellement, depuis le grec eran, agapan, philein, jusqu'à l'anglais to love, to like, et pour lequel il faudra trouver un terme générique correspondant dans la langue d'arrivée - un, ou plusieurs? -, qui permette à l'article de déployer l'histoire et la géographie de l'ensemble de ces terminologies. En revanche, « Nostalgie », mot français venu du grec via le suisse alémanique, est idiomatique, il est "en français » comme saudade est en portugais, Sehnsucht en allemand et dor en roumain. Ce premier travail de discrimination et de tri, qui impose tantôt de garder l'entrée française, tantôt de passer le lemme dans l'autre langue, n'est pas un banal travail éditorial de traduction, mais un travail de pensée lié à la traduction.

Le choix du ou des mots qui serviront dans une langue donnée d'équivalents pour les entrées génériques constitue un second problème philosophique, emblématique de cette non-superposabilité des langues et des réseaux qui fait l'objet même du Vocabulaire. Ce choix des lemmes est à son tour un simple miroir grossissant des difficultés et des dilemmes qu'il faut résoudre langue par langue, en particulier au moment des citations (notamment d'auteurs de l'antiquité) pour lesquelles les traductions existantes révèlent leurs insuffisances.

16 A partir de ces difficultés "techniques", chaque traduction se révèle être une adaptation et une aventure. Elle élabore ses stratégies, et réfléchit sur les effets qu'elle veut produire. Elle procède à des transformations fortes. Ce sont, en un second temps, ces transformations que nous voulons comparer : prendre au sérieux la différence des langues et des cultures, la mettre en lumière et l'interroger au moyen de ce dispositif de transfert, qui redouble la question de la traduction, oblige à une réflexion critique sur la pratique et constitue un outil puissant d'interrogation comparative.

L'enjeu, en effet, n'est pas identique pour chacune des langues. Chaque traduction en langue fixe une terminologie. Or cette terminologie est aujourd'hui plus ou moins flottante, pour des raisons non seulement culturelles, mais aussi historiques et politiques, interférant avec le sentiment national. Tel est le cas, en particulier, en Ukraine : il s'agit de constituer une langue philosophique propre, distincte du russe, en 
faisant travailler ensemble toute une communauté de philosophes, enseignants et chercheurs, qui s'ignorait comme telle. De même en roumain, où il s'agit de jouer entre plusieurs traditions dominantes et plusieurs espaces culturels, à travers une négociation entre latin et slavon. Avec le portugais et l'espagnol, il y va des frontières entre littérature et philosophie, mais aussi du rapport aux transformations des langues mères par les langues indigènes: il n'est certes pas indifférent à la géopolitique philosophique que l'espagnol se fasse au Mexique, que le portugais se fasse au Brésil ni que l'anglais soit de l'américain. Dans le monde anglophone, il ne va pas de soi de donner droit de cité à la différence des langues en philosophie, là où une certaine philosophie analytique supposerait plutôt des concepts indépendants des mots pour les dire et nécessairement peu situés dans l'espace et dans le temps. Ainsi le Dictionary of untranslatable terms qui va paraître à Princeton en 2013-2014 est-il pensé comme une machine de guerre contre le globish («se servir de l'english contre le globish») et contre la conception d'une philosophie trop rapidement universaliste indifférente à l'histoire et aux langues. Enfin, pour le monde arabe, l'enjeu est massif puisqu'il consiste à ouvrir l'une à l'autre des langues et des cultures que l'histoire a certes déjà réunies - en témoigne d'ailleurs la présence dans le Vocabulaire de l'arabe comme langue de passage et vecteur de transmission philosophique -, mais qui depuis lors se sont très largement ignorées comme l'atteste le très petit nombre de traductions modernes vers l'arabe jusqu'à aujourd'hui; la traduction en arabe littéral participe du nouveau moment d'accélération historique dans l'arrivée des textes, après celui du IXe et celui du XIXe siècle, et s'appuie sur le système de la langue arabe pour créer de nouveaux paronymes, contribuant à redessiner les frontières du référentiel intellectuel. Quant à la traduction iranienne qui se met en place, son importance politique se passe de commentaire.

Chaque traduction est ainsi l'occasion de transformations et d'ajouts parfois substantiels : nouvel article dans la traduction brésilienne sur la transformation du portugais par les langues indigènes, nouvel article sari'a dans la traduction arabe, nouvel article gender dans la traduction américaine, nouvel article sur « Le vocabulaire philosophique roumain (ancien, moderne, contemporain) » et nombreux encadrés etc.

\section{Intraduisibles.org : de la traduction assistée par ordinateur}

Si l'objectif à court et moyen terme est de réaliser au mieux la traduction du Vocabulaire en chacune des langues considérées, l'objectif est aussi, à plus long terme, de visualiser et de penser le géométral de toutes les réfections et de toutes les adaptations auxquelles l'opération de traduction et de transfert aura contraint. Il faudrait rassembler les singularités de chacune des versions, leurs ajouts et leurs transformations par rapport à l'« original » français, et les publier en français (d'abord et en tout cas, mais anagkê stênai) comme un volume en soi, guide pour nous des détours $\mathrm{du}$ «Philosopher en langues». Nous avons pour ce faire développé simultanément à partir de la France et à partir du Brésil, un site plurilingue qui permet, dans la partie réservée, d'échanger les questions et les traductions, notamment quant aux citations (www.intraduisibles.org/). Nous avons également élaboré au long cours un "Journal de bord des traductions ", publié en quatre langues, dont l'arabe et le turc, par la revue en ligne Transeuropéennes (www.transeuropeennes.eu/) 
20 Nous réfléchissons aux modalités permettant la numérisation-consultation la plus intelligente du Vocabulaire et de ses versions-adaptations, et autorisant l'ouverture raisonnée aux ajouts. Il y a là en effet quelque chose de conforme à l'esprit de ce travail. D'une part, parce que le Vocabulaire est un geste plutôt qu'une œuvre close (une energeia plutôt qu'un ergon, nous ne cessons de répéter la manière dont Humboldt caractérise la langue); si bien que l'ajout de symptômes, langue par langue, va évidemment dans le sens de la démarche, à condition de garder un certain contrôle sur les ajouts en question. D'autre part, parce que ces ajouts ont eux-mêmes vocation à entrer en résonance entre eux, pour constituer visiblement un maillage comparatif des traditions et des cultures philosophiques.

21 Un tel réseau prend pour point d'ancrage, donc pour " mots-clefs ", non seulement les lemmes analogues dans les différentes langues, mais les citations (et/ ou leur référence) autour desquelles se constituent les articles, de manière à les faire apparaître à la fois dans leur langue originale et dans leurs traductions, multiples dans l'espace et dans le temps. Nous poursuivons ainsi le travail initié lors de la participation au projet ECHO (European Cultural Heritage Online, piloté par le Max Planck Institut, lors du 5ème PCRD) et qui a donné lieu à un prototype de numérisation intelligente, avec représentation cartographique des rapports entre entrées, navigation hypertexte, ensemble de liens externes, via mots-clefs, noms propres et citations, permettant d'accéder aux œuvres en langues (échantillon consultable également sur le site du Max Planck et sur celui du Robert [http://robert.bvdep.com/public/vep/accueil.html], voir en particulier l'article «Bild»). Nous voulons étendre cet échantillon, qui porte sur le vocabulaire de l'image à partir du dictionnaire français, aux adaptations des mêmes entrées dans certaines autres langues, afin de comparer les cartographies et d'implémenter la navigation interne et externe.

Une telle recherche ouvre, à plus long terme, sur une réflexion quant aux modèles à l'œuvre dans la traduction automatique.

23 Le principal modèle à l'œuvre jusqu'à aujourd'hui, lié à Systran, consiste à faire passer d'une langue à l'autre via une langue-pivot, l'anglais, qui fonctionne comme commun dénominateur. L'anglais est lui-même préalablement désambigué (essentiellement via Wordnet), donc transformé pour passer du statut de langue naturelle à celui de languepivot. La désambiguation est comprise comme le moyen de passer du mot, singulier et éclairé par la langue, au concept universel. Cette conception est celle d'une bonne partie de la tradition philosophique, depuis Aristote qui construit l'homonymie comme le mal radical du langage, jusqu'à Leibniz dont la caractéristique universelle vise une réduction aux identiques permettant des opérations de calcul. Traduire consiste alors à ramener toutes les langues naturelles à une unique langue conceptuelle neutre, sans qualités, autorisant comme un échangeur un nouveau passage à une quelconque autre langue naturelle. Dans cette perspective, la différence entre les langues naturelles est accidentelle et réductible.

24 En partant du Vocabulaire et de ses traductions, nous voudrions explorer la possibilité d'un modèle inverse: affronter et exploiter la pluralité au lieu de viser l'unité. La comparaison requiert non pas un tertium quid commun (un langage conceptuel, "globish-technish»), mais un espace ou une géométrie commune, une topique, une topologie, permettant de montrer en quoi les réseaux terminologiques sont et en quoi ils ne sont pas superposables d'une langue à l'autre, et même d'une œuvre à l'autre au sein d'une même langue (époque, genre, auteur, style); en quoi, de manière analogue, 
les syntaxes sont et en quoi elles ne sont pas superposables. Une réévaluation de l'homonymie, comme caractéristique d'une langue naturelle comme telle, constitue une clef. La phrase déjà citée de Lacan, dans L'Etourdit, peut servir de repère : «Une langue, entre autres, n'est rien de plus que l'intégrale des équivoques que son histoire y a laissé subsister ». Le recensement des équivoques constitue, comme pour le semantic web, un point de passage obligé. Mais la manière de les traiter diffère considérablement: un certain nombre d'entre elles, à chaque fois différentes, sont constitutives d'une langue, elles sont non accidentelles et évoluent diachroniquement, enfin elles sont surtout visibles de l'extérieur de cette langue. Ainsi c'est pour «nous » (quel «nous»?) que le russe pravda signifie de manière équivoque "vérité » et "justice», ou svet «lumière » et «monde ». Inversement, notre "vérité » est un homonyme du point de vue slave, puisque le terme écrase "pravda», qui relève de la justice, et «istina", qui relève de l'être et de l'exactitude. On réfléchirait de même à l'ambiguïté pour «nous » de la racine «svet », lumière/monde, et à la problématique homonymie de «mir», paix, monde et commune paysanne, sur laquelle ne cesse de jouer Tolstoï. De même, c'est pour un latin que le grec logos signifie simultanément ratio et oratio, et pour un large "nous " que l'espagnol ser et l'espagnol estar sont non différenciés. C'est évidemment un ensemble langue-tradition-culture qui se retrouve ainsi interrogé.

Les suppositions épistémologiques diffèrent alors : on ne traite pas de concepts, mais de mots, c'est-à-dire de mots en langues, et sans doute de mots fortement contextualisés, pris dans des œuvres et des textes (problème global/local) On aboutit à un faisceau de questions : comment formaliser la description de la «richesse " homonymique d'un mot, d'une expression, d'une phrase? Comment formaliser le rapport synonymique entre deux «richesses" ? Comment modéliser le tracé des réseaux et rendre visible leur non-superposition? Peut-on modéliser le passage d'un nuage d'homonymes à un nuage d'homonymes? Ces questions recoupent celle du traitement des occurrences et des contextes qui, sans que l'idéologie de la traduction ait changé, commencent à infléchir le modèle de la langue-pivot. Elles sont à l'horizon de nos recherches.

\section{INDEX}

Mots-clés : dictionnaire, traduire, langue, globish

Schlüsselwörter : Wörterbuch, übersetzen, Sprache, globish

\section{AUTEUR}

\section{BARBARA CASSIN}

Barbara Cassin est directrice de recherches au CNRS. Pour plus d'informations, voir la notice suivante. 